\title{
FATORES ASSOCIADOS À OCORRÊNCIA DE LESÕES DURANTE A PRÁTICA DE ATIVIDADE FÍSICA EM ACADEMIAS AO AR LIVRE
}

\author{
CORRELATES TO INJURIES DURING PHYSICAL ACTIVITY PRACTICE AT FITNESS ZONES
}

Artigo Original

Original ARTICLE

Artículo Original

\author{
FACTORES ASOCIADOS A LA APARICIÓN DE LESIONES DURANTE ACTIVIDAD FISICA \\ EN GIMNASIOS AL AIRE LIBRE
}

\begin{abstract}
Alice Tatiane da Silva ${ }^{1,2}$
(Educadora Física)

Rogério César Fermino 2,3

(Educador Físico)

Claudia Oliveira Alberico 2,4

(Educadora Física)

Rodrigo Siqueira Reis 1,2,5

(Educador Físico)
\end{abstract}

\section{Universidade Federal do Paraná, Programa de Pós-Graduação em Educação Física. Curitiba, PR, Brasil. 2. Pontifícia Universidade Católica do Paraná, Grupo de Pesquisa em Atividade Física e Qualidade de Vida (GPAQ/PUCPR). Curitiba, PR, Brasil. \\ 3. Universidade Tecnológica Federal do Paraná, Programa de Pós- \\ Graduação em Educação Física. Curitiba, PR, Brasil. \\ 4. Centro Universitário Campos de Andrade, Curso de Educação Física. Curitiba, PR, Brasil. \\ 5. Washington University in St Louis, Brown School, Prevention Research Center. St Louis-MO, United States of America.}

\section{Correspondência:}

Grupo de Pesquisa em Atividade Física e Saúde. Curso de Educação Física - PUCPR. Rua Imaculada Conceição, 1.155, Prado Velho, Curitiba, PR, Brasil. 80215-90. silva.alice@outlook.com

\section{RESUMO}

Introdução: Evidências apontam associação positiva entre a prática de atividades físicas e a ocorrência de lesões. No entanto, faltam estudos que tenham analisado essa ocorrência relacionada com as academias ao ar livre (AAL). Objetivo: Verificar os fatores associados à ocorrência de lesões durante a prática de atividades físicas nas academias ao ar livre em adultos de Curitiba-PR. Métodos: Estudo transversal realizado em 2012, no qual foram entrevistados "in loco" 411 frequentadores (53,8\% mulheres) de 20 AAL da cidade. A ocorrência de lesões foi autorreportada e a sua associação foi testada com variáveis individuais e sociodemográficas (sexo, faixa etária, situação conjugal, escolaridade, renda domiciliar, índice de massa corporal), prática de atividade física no lazer (caminhada e atividade física total) e características de uso das AAL (percepção de esforço, frequência semanal, tempo de permanência, meses de uso e orientação) com a regressão de Poisson no software STATA 12. Resultados: A ocorrência de lesões foi de $3,4 \%$ e as variáveis não foram associadas ao desfecho. Conclusão: A ocorrência de lesões é baixa e a prática de atividade física nesses equipamentos aparenta ser segura para a integridade física dos seus frequentadores.

Descritores: atividade motora; traumatismos em atletas; academias de ginástica; estudos epidemiológicos; estudos transversais.

\begin{abstract}
Introduction: Evidence suggests that there is a positive association between physical activity and injuries. However, few studies have examined this association in connection with the use of outdoor fitness zones (FZ). Objective: To determine the association between injuries and physical activity among adults who use Fitness Zones (FZ) in the city of Curitiba, PR, Brazil. Methods: A cross-sectional study was conducted in 2012, which involved interviews with 411 users (53.8\% women) in loco at twenty FZ in Curitiba. Injuries were self-reported, and their association was tested with individual and sociodemographic variables (sex, age, marital status, schooling, household income, body mass index), leisure physical activity (walking and total physical activity) and characteristics of use of the FZ (perceived exertion, weekly frequency, time spent at the FZ, months of use, and guidance given). Poisson regression was applied, using the software STATA 12. Results: The rate of injuries was $3.4 \%$ and the variables tested were not associated with the outcome. Conclusion: The occurrence of injuries due to use of the FZ is low, and the physical activity conducted on this equipment appears to be safe for users.
\end{abstract}

Keywords: motor activity; athletic injuries; fitness centers; epidemiologic studies; cross-sectional studies.

\section{RESUMEN}

Introducción: La evidencia sugiere una asociación positiva entre la actividad física y la aparición de lesiones. Sin embargo, hay pocos estudios que han examinado esta ocurrencia relacionada con gimnasios al aire libre (GAL). Objetivo: Evaluar los factores asociados con la aparición de lesiones durante la actividad física en gimnasios al aire libre (GAL) para adultos en Curitiba-PR. Métodos: Estudio transversal en el año 2012, en el que se entrevistaron 411 usuários "in loco" (53,8\% mujeres) en 20 GAL de la ciudad. La aparición de lesiones fue evaluada por el auto-reporte y la asociación se puso a prueb a con variables individuales y sociodemográficas (sexo, edad, estado civil, educación, ingreso familiar, índice de masa corporal), la actividad física en el tiempo libre (caminar y actividad física total) y las características de uso de GAL (esfuerzo percibido, frecuencia semanal, duración de la estancia, meses de uso y orientación) con la regresión de Poisson en el software STATA 12. Resultados: La incidencia de lesiones fue de 3,4\% y las variables no se asociaron con el resultado. Conclusión: La incidencia de lesiones es baja y ese tipo de equipo parece ser seguro para la integridad física de sus visitantes habituales.

Descriptores: actividad motora; traumatismos en atletas; centros de acondicionamiento; estudios epidemiológicos; estudios transversales. 


\section{INTRODUÇÃO}

Os programas para a promoção da prática de atividade física têm sido implementados com o objetivo de melhorar a saúde e a qualidade de vida da população ${ }^{1-3}$. Apesar dos inúmeros benefícios associados, alguns riscos são inerentes a esta prática, entre eles, a ocorrência de lesões osteomioarticulares e tendíneas ${ }^{1,4}$. Portanto, medidas que atenuem a exposição ao risco destas lesões poderiam ser realizadas, como por exemplo, a supervisão das atividades por um profissional de Educação Física ${ }^{5}$, assim como aumentar o conhecimento da população sobre os fatores de risco, entre outros ${ }^{6}$.

Ao longo dos últimos anos, diversas cidades têm disponibilizado equipamentos para a prática de atividades físicas em locais públi$\cos ^{7-9}$. Em Curitiba-PR, desde 2008, a prefeitura iniciou a instalação de "academias ao ar livre" (AAL) ${ }^{10}$, as quais são compostas por aparelhos tubulares em aço em que as pessoas podem realizar atividades aeróbicas, exercícios resistidos, de alongamento e de mobilidade articular, porém sem a orientação e acompanhamento de um profissional de Educação Física ${ }^{11,12}$. Placas informativas sobre o uso dos equipamentos estão disponíveis nas $A \mathrm{AL}^{12}$, mas apenas a disponibilidade destas poderiam ser insuficientes para atenuar a exposição ao risco de lesões ${ }^{6}$. Em geral, duas em cada dez pessoas reportam lesões em decorrência da prática de atividade física no tempo de lazer ${ }^{13,14}$, a qual pode aumentar de acordo com as características inerentes à atividade, como intensidade, volume e complexidade das ações motoras ${ }^{15-20}$. Além disso, sexo masculino, menor idade e maior aptidão cardiorrespiratória estão positivamente associados com a ocorrência das lesões ${ }^{14,21}$.

Os aparelhos das AAL apresentam baixa complexidade de execução dos movimentos, mas em alguns, a carga pode variar de acordo a massa corporal do praticante ${ }^{12}$. Assim, a falta de supervisão direta poderia, em alguma medida, aumentar a exposição ao risco de lesões ${ }^{6}$. Desconhecem-se estudos que tenham identificado a ocorrência de lesões durante a prática de atividades físicas nas AAL e quais características estariam associadas a este agravo de saúde.

O objetivo deste estudo foi verificar os fatores associados com a ocorrência de lesões durante a prática de atividades físicas nas AAL em adultos de Curitiba-PR.

\section{MATERIAIS E MÉTODOS}

Os dados deste estudo fazem parte do projeto "Academias ao ar livre de Curitiba: efeitos sobre o nível de atividade física e qualidade de vida da população'11 . Durante o segundo semestre de 2012, foi realizado um estudo exploratório com delineamento transversal e aplicação de entrevistas face-a-face "in loco" em que participaram adultos frequentadores de $20 \mathrm{AAL}$ de Curitiba. O projeto foi aprovado pelo Comitê de Ética em Pesquisa da PUCPR (85033/2012) e os participantes assinaram um Termo de Consentimento Livre e Esclarecido.

Até o mês de julho de 2016, Curitiba contava com 196 AAL implantadas em diferentes bairros ${ }^{10}$. Mas, no início do planejamento da pesquisa (julho de 2012), a cidade possuía $100 \mathrm{AAL}^{10,11}$. Informações detalhadas sobre o processo de amostragem representativa e seleção das 20 AAL podem ser consultadas na literatura ${ }^{11}$

\section{Procedimentos utilizados para a coleta de dados}

Quinze entrevistadores, integrantes do grupo de pesquisa e alunos de graduação e pós-graduação, realizaram treinamento teórico-prático de oito horas sobre os critérios de seleção, inclusão, abordagem dos participantes, condução das entrevistas, preenchimento dos formulários e identificação da taxa de recusa. Todos foram instruídos a abordarem intencionalmente o maior número de adultos que estivessem na área das AAL praticando atividade física. Caso a AAL estivesse vazia, os entrevistadores deveriam permanecer durante todo o tempo previsto no local (60 minutos) na tentativa de abordar algum frequentador.

Em cada AAL a coleta ocorreu em quatro dias, incluindo dois dias de semana e dois dias de final de semana (segunda-feira, quinta-feira, sábado e domingo) e em quatro períodos do dia (8-9h, 11-12h, 14-15h e 17-18h). Este procedimento foi adotado com o objetivo de representar diferentes perfis de frequentadores. A coleta teve duração de 49 dias (outubro a dezembro de 2012) com temperatura média de $22^{\circ} \mathrm{C}\left(15-30^{\circ} \mathrm{C}\right)^{11}$.

\section{Variável dependente}

A ocorrência de lesões foi avaliada com a questão: "Alguma vez você já se machucou ou se lesionou praticando atividade física nos equipamentos das AAL?" ("não"/"sim"). Esta medida autorreportada é comumente utilizada em pesquisas similares que verificaram a ocorrência de lesões decorrentes da prática de atividades físicas ${ }^{14,17}$.

\section{Variáveis independentes}

O sexo foi observado, a idade classificada em duas faixas etárias (18-59 anos e $\geq 60$ anos) e a situação conjugal categorizada em "solteiro" (solteiro, separado, viúvo) ou "casado" (casado, vivendo com parceiro). A escolaridade completa foi categorizada em "até ensino médio"e "ensino superior" e a renda domiciliar mensal agrupada em " $\mathrm{R} \$ \leq 2.999,00$ " e " $\geq R \$ 3.000,00 "$. O índice de massa corporal (IMC) foi obtido com informações de massa corporal e estatura autorreportadas e os participantes classificados em IMC "até $24,9 \mathrm{~kg} / \mathrm{m}^{2 "}$ e " $\geq 25,0 \mathrm{~kg} / \mathrm{m}^{2}$ ".

A atividade física foi avaliada com o módulo de lazer do International Physical Activity Questionnaire (IPAQ22, traduzido, adaptado e validado para a utilização no Brasil ${ }^{23}$. Os entrevistados reportaram a frequência semanal e o tempo despendido na prática de caminhada e de atividades físicas de intensidades moderadas e vigorosas em uma semana habitual. Os escores de caminhada e de atividade física total no tempo de lazer foram categorizados em "0-149 min/sem" e " $\geq 150 \mathrm{~min} / \mathrm{sem}^{\prime}$. As informações do IPAQ referiam-se as atividades de lazer, desconsiderando aquelas realizadas nas AAL.

A percepção de esforço realizado nas AAL foi avaliada com a questão: "Como você percebe o seu esforço físico nos equipamentos desta AAL?" e classificada em "leve" e "médio/forte". A frequência de uso dos locais foi avaliada com a questão: "Com que frequência você vem a esta AAL?", e a resposta classificada em " $\leq 2$ vez/sem" e " $\geq 3$ vez/sem". O tempo de permanência foi avaliado com a questão: "Em um dia normal que você vem à $A A L$, quanto tempo você fica neste local?" e classificado em " $\leq 30 \mathrm{~min} /$ dia" e $^{\prime} \geq 31 \mathrm{~min} /$ dia". A quantidade de meses de uso dos equipamentos foi avaliada com a questão: "Há quanto tempo você frequenta esta AAL?" e categorizado em " $\leq 11$ meses" e " $\geq 12$ meses".

Por fim, a orientação para o uso das AAL foi avaliada com a questão: "Você teve alguma orientação sobre como utilizar os aparelhos da AAL?". Os participantes poderiam relatar a orientação proveniente de diferentes fontes (placas, amigos, médico, profissional da prefeitura e/ou Educação Física, outros meios). Esta variável foi categorizada em "não" e "sim".

\section{Análise estatística}

Os dados foram analisados com a estatística descritiva e distribuição de frequências absoluta e relativa. A ocorrência de lesões foi comparada entre as categorias das variáveis individuais, sociodemográficas, prática de atividade física e de uso das AAL. As associações foram testadas com a regressão de Poisson ${ }^{24}$ no software STATA 12 e o nível de significância mantido em $5 \%$. 


\section{RESULTADOS}

Foram entrevistados 411 frequentadores (53,8\% mulheres) e a taxa de recusa para a participação na pesquisa foi de 12,9\% ( $n=88)$ (Tabela 1). A maior parte dos frequentadores estava na faixa etária entre 18-59 anos $(65,0 \%)$, eram casados $(65,9 \%)$, com ensino superior completo $(53,4 \%)$, de renda domiciliar mensal $\leq R \$ 2.999,00$ (62,5\%) e com IMC $\geq 25,0$ kg/ $m^{2}(60,9 \%)$ (Tabela 1). Em relação a prática de atividades físicas, 55\% dos entrevistados realizavam menos de $150 \mathrm{~min} / \mathrm{sem}$ de caminhada, enquanto que 72,9\% realizam mais de $150 \mathrm{~min} / \mathrm{sem}$ de atividade física total no tempo de lazer. Maior proporção dos entrevistados relatou a percepção de esforço realizado nos equipamentos da AAL como médio/forte (77,4\%). Cerca de sete a cada 10 participantes relataram frequentar as $A A L \geq 3$ vez/sem (68,8\%), enquanto cinco a cada 10 entrevistados reportaram permanecer no local $\leq 30 \mathrm{~min} / \mathrm{dia}(50,7 \%) \mathrm{e}$ frequentavam a AAL por um período $\leq 11$ meses (52,3\%). A maior parte dos entrevistados $(71,0 \%)$ relatou ter recebido algum tipo de orientação para o uso dos equipamentos (Tabela 1).

A ocorrência de lesões durante a prática de atividades físicas nas AAL foi de 3,4\% ( $n=13$ ) (Tabela 1) e nenhuma das variáveis testadas apresentou associação significante com o desfecho (Tabela 2). No entanto, a ocorrência de lesões foi superior nos participantes com as seguintes características: sexo feminino (4,0\%), faixa etária entre 1859 anos (3,7\%), casados (3,9\%), ensino superior completo (4,7\%), IMC $\leq 24,9 \mathrm{~kg} / \mathrm{m}^{2}(4,8 \%), \geq 150 \mathrm{~min} / \mathrm{sem}$ de caminhada $(4,1 \%)$ ou de atividade

Tabela 1. Características descritivas dos frequentadores das academias ao ar livre (AAL). Curitiba-PR, $2012(\mathrm{n}=411)$.

\begin{tabular}{|c|c|c|c|}
\hline Variáveis & Categoria & $n$ & $\%$ \\
\hline \multicolumn{4}{|l|}{ Individuais e sociodemográficas } \\
\hline \multirow{2}{*}{ Sexo } & Masculino & 190 & 46,2 \\
\hline & Feminino & 221 & 53,8 \\
\hline \multirow{2}{*}{ Faixa etária (anos) } & $18-59$ & 267 & 65,0 \\
\hline & $\geq 60$ & 144 & 35,0 \\
\hline \multirow{2}{*}{ Situação conjugala } & Solteiro & 140 & 34,1 \\
\hline & Casado & 270 & 65,9 \\
\hline \multirow{2}{*}{ Escolaridade completa ${ }^{a}$} & Até o ensino médio & 139 & 46,6 \\
\hline & Ensino superior & 159 & 53,4 \\
\hline \multirow{2}{*}{ Renda domiciliar mensal (R\$) ${ }^{a}$} & $\leq 2.999,00$ & 225 & 62,5 \\
\hline & $\geq 3.000,00$ & 135 & 37,5 \\
\hline \multirow{2}{*}{ Índice de massa corporal $\left(\mathrm{kg} / \mathrm{m}^{2}\right)^{\mathrm{a}}$} & $\leq 24,9$ & 157 & 39,1 \\
\hline & $\geq 25,0$ & 245 & 60,9 \\
\hline \multicolumn{4}{|l|}{ Prática de atividade física no tempo de lazer } \\
\hline \multirow{2}{*}{ Caminhada (min/sem) } & $0-149$ & 226 & 55,0 \\
\hline & $\geq 150$ & 185 & 45,0 \\
\hline \multirow{2}{*}{ Atividade física total ${ }^{*}(\mathrm{~min} / \mathrm{sem})^{a}$} & $0-149$ & 111 & 27,1 \\
\hline & $\geq 150$ & 299 & 72,9 \\
\hline \multicolumn{4}{|l|}{ Uso das AAL } \\
\hline \multirow{2}{*}{$\begin{array}{l}\text { Percepção de esforço durante a prática } \\
\text { de exercícios na } A A L^{a}\end{array}$} & Leve & 86 & 22,6 \\
\hline & Médio/forte & 294 & 77,4 \\
\hline \multirow{2}{*}{ Frequência semanal de uso das $\mathrm{AAL}^{\mathrm{a}}$} & $\leq 2 \mathrm{vez} / \mathrm{sem}$ & 119 & 31,2 \\
\hline & $\geq 3 \mathrm{vez} / \mathrm{sem}$ & 262 & 68,8 \\
\hline \multirow{2}{*}{ Tempo de permanência nas $A_{A} L^{a}$} & $\leq 30 \mathrm{~min} / \mathrm{dia}$ & 192 & 50,7 \\
\hline & $\geq 31 \mathrm{~min} / \mathrm{dia}$ & 187 & 49,3 \\
\hline \multirow{2}{*}{ Quantidade de meses de uso das AAL } & $\leq 11$ meses & 215 & 52,3 \\
\hline & $\geq 12$ meses & 196 & 47,7 \\
\hline \multirow{2}{*}{$\begin{array}{l}\text { Orientação para uso dos equipamentos } \\
\text { das AAL }\end{array}$} & Não & 119 & 29,0 \\
\hline & $\operatorname{sim}$ & 292 & 71,0 \\
\hline \multirow{2}{*}{$\begin{array}{l}\text { Ocorrência de lesões durante a prática } \\
\text { de atividades físicas nas } A A L^{a}\end{array}$} & Não & 367 & 96,6 \\
\hline & Sim & 13 & 3,4 \\
\hline
\end{tabular}

variáveis com valores ausentes. * $\mathrm{min} / \mathrm{sem}$ de caminhada + min/sem de atividade física moderada + min/ sem de atividade física vigorosa ( 2 ). física total no tempo de lazer (3,6\%), percepção de esforço entre médio/ forte $(3,7 \%)$, frequentar os locais $\geq 3$ vez/sem (3,8\%), permanecer nos locais $\leq 30 \mathrm{~min} /$ dia (4,7\%), utilizar as $A A L \geq 12$ meses (4,1\%), não receber orientação para o uso dos equipamentos (4,8\%) (Tabela 2).

Tabela 2. Associação bivariada entre as variáveis individuais, sociodemográficas, prática de atividade física e de uso das academias ao ar livre (AAL) com a ocorrência de lesões em adultos. Curitiba-PR, 2012 ( $n=411$ ).

\begin{tabular}{|c|c|c|c|c|c|c|}
\hline Variáveis & Categoria & $\mathrm{n}$ & $\%$ & $\mathrm{RP}$ & $\mathrm{IC}_{95 \%}$ & $p$ \\
\hline \multicolumn{7}{|l|}{$\begin{array}{c}\text { Individuais e } \\
\text { sociodemográficas }\end{array}$} \\
\hline \multirow[t]{2}{*}{ Sexo } & Masculino & 5 & 2,8 & 1 & & \\
\hline & Feminino & 8 & 4,0 & 1,42 & $0,47-4,36$ & 0,535 \\
\hline \multirow[t]{2}{*}{ Faixa etária (anos) } & $18-59$ & 9 & 3,7 & 1 & & \\
\hline & $\geq 60$ & 4 & 2,9 & 0,77 & $0,24-2,50$ & 0,665 \\
\hline \multirow[t]{2}{*}{ Situação conjugal } & Solteiro & 3 & 2,4 & 1 & & \\
\hline & Casado & 10 & 3,9 & 1,60 & $0,47-5,82$ & 0,470 \\
\hline \multirow[t]{2}{*}{ Escolaridade completa } & $\begin{array}{l}\text { Até ensino } \\
\text { médio }\end{array}$ & 2 & 1,6 & 1 & & \\
\hline & $\begin{array}{l}\text { Ensino } \\
\text { superior }\end{array}$ & 7 & 4,7 & 2,96 & $0,61-14,23$ & 0,176 \\
\hline \multirow[t]{2}{*}{ Renda domiciliar (R\$/mês) } & $\leq 2.999,00$ & 6 & 3,0 & 1 & & \\
\hline & $\geq 3.000,00$ & 4 & 3,0 & 1,02 & 0,29-3,61 & 0,975 \\
\hline \multirow[t]{2}{*}{$\begin{array}{l}\text { Índice de massa corporal } \\
\left(\mathrm{kg} / \mathrm{m}^{2}\right)\end{array}$} & $\leq 24,9$ & 7 & 4,8 & 1 & & \\
\hline & $\geq 25,0$ & 6 & 2,7 & 0,55 & $0,18-1,64$ & 0,282 \\
\hline
\end{tabular}

\section{Prática de atividade física}

no tempo de lazer

\begin{tabular}{c|c|c|c|c|c|c}
\hline Caminhada (min/sem) & $0-149$ & 6 & 2,9 & 1 & & \\
\cline { 2 - 8 } & $\geq 150$ & 7 & 4,1 & 1,44 & $0,48-4,29$ & 0,511 \\
\hline $\begin{array}{c}\text { Atividade física total* } \\
\text { (min/sem) }\end{array}$ & $0-149$ & 3 & 2,9 & 1 & & \\
\cline { 2 - 7 } & $\geq 150$ & 10 & 3,6 & 1,23 & $0,34-4,46$ & 0,756 \\
\hline Uso das AAL
\end{tabular}

\begin{tabular}{c|c|c|c|c|c|c}
\hline Percepção de esforço durante & Leve & 2 & 2,3 & 1 & & \\
\cline { 2 - 8 } a prática de exercícios na AAL & Médio/forte & 11 & 3,7 & 1,61 & $0,37-7,26$ & 0,536 \\
\hline \multirow{2}{*}{$\begin{array}{c}\text { Frequência semanal de } \\
\text { uso das AAL }\end{array}$} & $\leq 2$ vez/sem & 3 & 2,5 & 1 & & \\
\cline { 2 - 8 } & $\geq 3$ vez/sem & 10 & 3,8 & 1,52 & $0,42-5,54$ & 0,521 \\
\hline \multirow{2}{*}{$\begin{array}{c}\text { Tempo de permanência } \\
\text { nas AAL }\end{array}$} & $\leq 30 \mathrm{~min} / \mathrm{dia}$ & 9 & 4,7 & 1 & & \\
\cline { 2 - 8 } & $\geq 31 \mathrm{~min} / \mathrm{dia}$ & 4 & 2,1 & 0,46 & $0,14-1,15$ & 0,192 \\
\hline \multirow{2}{*}{$\begin{array}{c}\text { Quantidade de meses de } \\
\text { uso das AAL }\end{array}$} & $\leq 11$ meses & 5 & 2,7 & 1 & & \\
\cline { 2 - 8 } & $\geq 12$ meses & 8 & 4,1 & 1,50 & $0,49-4,60$ & 0,491 \\
\hline $\begin{array}{c}\text { Orientação para uso dos } \\
\text { equipamentos das AAL }\end{array}$ & Não & 5 & 4,8 & 1 & & \\
\cline { 2 - 7 } & Sim & 8 & 2,9 & 0,60 & $0,20-1,84$ & 0,375 \\
\hline
\end{tabular}

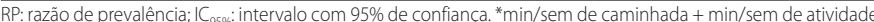
física moderada $+\mathrm{min} / \mathrm{sem}$ de atividade física vigorosa $\left({ }^{*} 2\right)$

\section{DISCUSSÃO}

Este estudo teve o objetivo de verificar os fatores associados com a ocorrência de lesões durante a prática de atividades físicas nas AAL em adultos. Tal ocorrência foi de 3,4\% e as variáveis testadas não apresentaram associação com o desfecho. Conhecer os possíveis fatores associados a este agravo de saúde é importante para identificar os grupos populacionais mais vulneráveis à ocorrência de lesões para que seja possível planejar intervenções efetivas para prevenir e reduzir tal ocorrência.

Revisando a literatura, constatou-se não existirem estudos que tenham identificado os fatores associados com as lesões durante a prática de atividades físicas nas AAL ou em equipamentos similares. Ainda que estas associações tenham sido testadas em diversos estudos que envolveram atividades esportivas ${ }^{13,14,16-20,25,26}$, as diferenças inerentes às atividades (finalidade, intensidade, complexidade da ação motora, 
uso de equipamentos específicos, entre outras) limitam, em parte, a adequada comparação dos resultados. Por exemplo, em adultos de Pelotas, RS, Brasil a prevalência de lesões em decorrência da prática de musculação, atividade que mais se aproxima àquelas realizadas nas $A A L$, foi de $19,4 \%^{14}$. No entanto, os autores não testaram as variáveis associadas com a prevalência de lesões específicas pela modalidade de atividade física ${ }^{14}$.

No presente estudo, a ocorrência de lesões (3,4\%) foi consideravelmente inferior ao reportado na literatura, em que a prevalência de lesões em decorrência da prática de atividades físicas no lazer é de $22 \%{ }^{13,14}$. Valores substancialmente superiores foram observados em esportes recreativos, amadores e de alto rendimento (29-100\%) ${ }^{15-17,19,20,25}$. A variabilidade nas prevalências de lesões reportadas entre as modalidades pode ser explicada por variáveis inerentes às atividades, uma vez que a frequência, a intensidade, o tempo e o tipo (F.I.T.T.) de algumas modalidades de atividade física apresentam maior potencial lesivo que outras 6,21,26-28.

As variáveis individuais de sexo e faixa etária não apresentaram associação com a ocorrência de lesões no presente estudo. No entanto, Rombaldi et al. ${ }^{14}$ verificaram que o sexo masculino e menor faixa etária foram associados com a ocorrência de lesões na atividade física em amostra representativa de adultos de Pelotas, RS, Brasil. Os autores justificam estas associações ao fato de homens e indivíduos mais jovens estarem engajados em atividades físicas de maior intensidade ${ }^{14}$. A ausência destas associações no presente estudo poderiam ser explicadas pelo fato dos indivíduos realizarem atividades físicas sistematizadas em equipamentos que apresentam complexidade similar, independente do sexo e faixa etária ${ }^{12}$, os quais poderiam minimizar o risco de lesões. No entanto, estudos que testaram os fatores associados com a ocorrência de lesões no esporte verificaram associação positiva com sexo masculino, maior IMC, menor faixa etária e maior intensidade do treinamento 15-17,19,20. É importante ressaltar que estas associações foram encontradas em praticantes de modalidades esportivas, o que difere substancialmente da população investigada neste estudo. Assim como na presente pesquisa, outras evidências não encontraram associação entre IMC ${ }^{14,21}$, situação conjugal ${ }^{14}$ e nível socioeconômico ${ }^{14}$ com a ocorrência de lesões em decorrência da prática de atividades físicas.

Não se conhecem estudos que tenham investigado a associação entre escolaridade e ocorrência de lesões. A literatura reporta que pessoas com maior grau de escolaridade tendem a ter maior acesso as informações sobre os cuidados gerais com a saúde, garantindo a mínima autonomia durante a prática de atividades físicas, principalmente quando esta é realizada sem a supervisão de um profissional de Educação Física ${ }^{6}$. Apesar de não apresentar associação significante, no presente estudo foi observado o oposto, onde os participantes com maior grau de escolaridade apresentaram maior ocorrência de lesões $(4,7$ versus 1,6\%).

Evidências apresentam associação positiva entre volume de treinamento $\geq 76 \mathrm{~min} / \mathrm{sem}$ com a ocorrência de lesões em corredores de rua e praticantes de esportes ${ }^{21}$. Ainda que cerca de $73 \%$ dos entrevistados tenham relatado praticar $\geq 150 \mathrm{~min} / \mathrm{sem}$ de atividade física com intensidade moderada e vigorosa, esta característica não foi associada com a ocorrência de lesões. Uma possível explicação seria o fato das atividades realizadas no tempo de lazer apresentarem caráter recreacional e de menor complexidade ${ }^{6}$. Assim como no presente estudo, Hootman et al. ${ }^{21}$ não encontraram associação significante entre a prática de caminhada e a ocorrência de lesões, sugerindo que esta atividade é segura e apresenta baixo potencial lesivo, quando comparado com as atividades esportivas ${ }^{14}$.

O tempo de utilização das AAL em meses não apresentou associação com a ocorrência de lesões. Evidências indicam que o tempo de experiência na prática de uma determinada atividade pode representar um fator de proteção para a ocorrência de lesões, apontando que a experiência torna os indivíduos aptos à execução das atividades ${ }^{16}$. Esta constatação poderia explicar os achados deste estudo, uma vez que cerca de $48 \%$ dos entrevistados frequentavam os locais há pelo menos 12 meses, e poderiam estar adaptados ao uso dos equipamentos das AAL.

Apesar da maioria dos entrevistados relatarem ter recebido algum tipo de orientação sobre o uso dos equipamentos da AAL (71\%), esta variável não esteve inversamente associada com a ocorrência de lesões. Ainda assim, como sugerido pela Sociedade Brasileira de Medicina do Esporte e Exercício ${ }^{5}$, seria importante proporcionar aos frequentadores dos locais a correta orientação e a supervisão das atividades nas AAL por um profissional de Educação Física, com o objetivo de melhorar a utilização destes equipamentos, minimizar o risco e evitar a ocorrência de lesões, evitando assim danos para a saúde daqueles que se exercitam no locais ${ }^{5,6}$.

Algumas limitações devem ser consideradas ao interpretar os resultados deste estudo. A baixa ocorrência de lesões pode ter sido resultado do viés de seleção, uma vez que foram entrevistados somente aquelas pessoas que estavam nas AAL realizando atividades físicas. Portanto, os indivíduos que possivelmente estivessem afastados da prática das atividades nos locais, em decorrência de lesões, não foram incluídos no inquérito. Ainda, devido a abordagem geral sobre a ocorrência das lesões, não foi possível identificar as características deste agravo (contusão, luxação, distensão, etc.). Por fim, o delineamento transversal do estudo não permite estabelecer a relação causal entre o desfecho e as variáveis testadas.

\section{CONCLUSÃO}

A ocorrência de lesões durante a prática de atividade física nas AAL é baixa e os fatores testados não foram associados a este desfecho. A utilização dos equipamentos das AAL para a prática de atividades físicas aparenta ser segura para a integridade física de seus frequentadores. Mesmo com a baixa ocorrência, as intervenções para prevenir as lesões poderiam ser direcionadas a todos os frequentadores, independente de sexo, faixa etária e demais características testadas no presente estudo, possibilitando assim, a utilização segura dos equipamentos pelas pessoas da comunidade.

Futuros estudos poderiam incluir amostra representativa dos moradores do entorno das AAL para verificar a prevalência de uso destes locais, assim como identificar a ocorrência de lesões entre os frequentadores não abordados nas AAL. Ainda, poderiam identificar o tipo, o local, o grau de severidade e o tempo de afastamento das atividades físicas em decorrência destas lesões.

\section{AGRADECIMENTOS}

Aos integrantes do Grupo de Pesquisa em Atividade Física e Qualidade de Vida (GPAQ/PUCPR) pela colaboração na coleta de dados e aos gestores da Secretaria Municipal de Esporte, Lazer e Juventude (SMELJ) por disponibilizar as informações sobre as AAL.

\section{FINANCIAMENTO}

ATS (orcid.org/0000-0002-9286-4345) recebeu bolsa da Coordenação de Aperfeiçoamento de Pessoal de Ensino Superior (CAPES) durante o mestrado. $O$ estudo foi parcialmente financiado pela Pontifícia Universidade Católica do Paraná (PUCPR).

Todos os autores declararam não haver qualquer potencial conflito de interesses referente a este artigo. 
CONTRIBUIÇÕES DOS AUTORES: Cada autor contribuiu individual e significativamente para o desenvolvimento do manuscrito. ATS (0000-0002-9286-4345)* participou da concepção inicial do estudo, revisão da literatura, análise dos dados e redação do manuscrito. RCF (0000-0002-9028-4179)* participou e coordenou a coleta de dados, concepção inicial do estudo, análise dos dados, revisão da literatura, além da redação e revisão crítica do manuscrito em todas as suas etapas. COA (00000001-8337-7978)* participou da coleta de dados, análise dos dados e colaborou na revisão do manuscrito. RSR (0000-0002-9872-9865)* foi responsável pela concepção do projeto, coordenou a coleta de dados e colaborou na revisão crítica do manuscrito. Todos os autores aprovaram a versão final do manuscrito e contribuíram com o conceito intelectual do estudo. *Número ORCID (Open Researcher and Contributor ID).

\section{REFERÊNCIAS}

1. Haskell WL, Lee IM, Pate RR, Powell KE, Blair SN, Franklin BA, Macera CA, Heath GW, Thompson PD, Bauman A. Physical activity and public health: updated recommendation for adults from the American College of Sports Medicine and the American Heart Association. Med Sci Sports Exerc. 2007;39(8):1423-34

2. Lee IM, Shiroma EJ, Lobelo F, Puska P, Blair SN, Katzmarzyk PT, et al. Effect of physical inactivity on major non-communicable diseases worldwide: an analysis of burden of disease and life expectancy. Lancet. 2012:380(9838):219-29.

3. Pucci GCMF, Rech CR, Fermino RC, Reis RS. Associação entre atividade física e qualidade de vida em adultos. Rev Saúde Públ. 2012;46(1):166-79

4. American College of Sports Medicine (ACSM). Diretrizes do ACSM para os testes de esforço e sua prescrição. 8a. ed. Rio de Janeiro: Guanabara; 2014

5. Sociedade Brasileira de Medicina do Esporte e Exercício (SBMEE). Nota da SBMEE sobre avaliação médica pré-participação no exercício e no esporte. Disponível em: http://www.medicinadoesporte. org.br. [Acesso em: 10 de março de 2013].

6. Simões NVN. Lesões desportivas em praticantes de atividade física: uma revisão bibliográfica. Rev Bras Fisioter. 2005;9(2):123-28

7. Fermino RC, Reis RC, Hallal PC, Kaczynski AT. Who are the users of urban parks? A study with adults from Curitiba, Brazil. J Phys Act Health. 2015;12(1):58-67.

8. Fermino RC, Reis RS, Cassou AC. Fatores individuais e ambientais associados ao uso de parques e praças por adultos de Curitiba-PR, Brasil. Rev Bras Cineantropom Desempenho Hum. 2012;14(4):377-89.

9. Hallal PC, Reis RS, Hino AAF, Santos MS, Grande D, Krempel MC, et al. Avaliação de programas comunitários de promoção de atividade física: o caso de Curitiba, Paraná. Rev Bras Ativ Fís Saúde. 2009;14(2):104-114.

10. Secretaria Municipal de Esporte, Lazer e Juventude (SMELJ). Academia ao ar livre. Disponível em: http:// www.curitiba.pr.gov.br/conteudo/academia-ao-ar-livre-smelj/144. [Acesso em: 25 de maio de 2015].

11. Souza CA, Fermino RC, Añez CRR, Reis RS. Perfil dos frequentadores e padrão de uso das academias ao ar livre em bairros de baixa e alta renda de Curitiba-PR. Rev Bras Ativ Fis Saúde. 2014;19(1):86-97.

12. Wendling NMS, Raumach R, Grande D, Kruchelski S, Cassou C, Mathoso E, et al. Prática e exercícios em academias ao ar livre. Rev Gestão Publ Curitiba. 2010;1(3):65-8.

13. Dekker R, Kingma J, Groothoff JW, Eisma WH, Ten Duis HJ. Measurement ofseverity of sports injuries: an epidemiological study. Clin Rehabil. 2000;14(6):651-6.

14. Rombaldi AJ, Silva MC, Barbosa MT, Pinto RC, Azevedo MR, Hallal PC, et al. Prevalência e fatores associados à ocorrência de lesões durante a prática de atividade física. Rev Bras Med Esporte. 2014;20(3):190-4
15. Grego LG, Monteiro HL, Padovani CR, Gonçalvez A. Lesões na dança: Estudo transversal híbrido em academias da cidade de Bauru-SP. Rev Bras Med Esporte. 1999;5(2):47-54

16. Hespanhol Jr LC, Costa LOP, Carvalho ACA, Lopes AD. Perfil das características do treinamento e associação com lesões musculoesqueléticas prévias em corredores recreacionais: um estudo transversal. Rev Bras Fisioter. 2012;16(1):46-53.

17. Hino AAF, Reis RS, Añez CRR, Fermino RC. Prevalência de lesões em corredores de rua e fatores associados. Rev Bras Med Esporte. 2009;15(1):36-9.

18. Hoshi RA, Pastre CM, Vanderlei LCM, Netto Jr J, Bastos FN. Lesões desportivas na ginástica artística: Estudo a partir de morbidade referida. Rev Bras Med Esporte. 2008;14(5):440-5.

19. Pastre CM, Carvalho Filho G, Monteiro HL, Netto Jr J, Padovani CR, García AB. Exploração dos fatores de risco para lesões no atletismo de alta performance. Rev Bras Med Esporte. 2007;13(3):200-4.

20. Souza JMC, Faim FT, Nakashima IY, Altruda CR, Medeiros WM, Silva LR. Lesões no karatê shotokan e no jiu-jitsu - trauma direto versus indireto. Rev Bras Med Esporte. 2011;17(2):107-10.

21. Hootman JM, Macera CA, Ainsworth BE, Martin M, Addy CL, Blair SN. Association among physical activity level, cardiorespiratory fitness, and risk of musculoskeletal injury. Am J Epidemiol. 2001;154(3):251-8.

22. Craig $C L$, Marshall AL, Sjöström M, Bauman AE, Booth ML, Ainsworth BE, et al. International physica activity questionnaire: 12-country reliability and validity. Med Sci Sports Exerc. 2003;35(8):1381-95.

23. Matsudo S, Araújo T, Matsudo V, Andrade D, Andrade E, Oliveira LC, et al. Questionário internacional de atividade física (IPAQ): Estudo de validade e reprodutibilidade no Brasil. Rev Bras Ativ Fís Saúde. 2001;6(2):5-18

24. Barros AJD, Hirakata VN. Alternatives for logistic regression in cross- sectional studies: an empirical comparison of models that directly estimate the prevalence ratio. BMC Med Res Methodol. 2003;3:21.

25. Guedes JM, Barbieri CF, Fabiane F. Lesões em tenistas competitivos. Rev Bras Ciênc Esporte 2010:31(3):217-29.

26. Zanuto EAC, Harada H, Gabriel Filho LRA. Análise epidemiológica de lesões e perfil físico de atletas do futebol amador na região do oeste paulista. Rev Bras Med Esporte. 2010;16(2):116-20.

27. Luciano PL, Lara LCR. Estudo epidemiológico das lesões do pé e tornozelo na prática desportiva recreacional. Acta Ortop Bras. 2012;20( 6):339-42.

28. Pileggi P, Gualano B, Souza M, Caparbo VF, Pereira RMR, Pinto ALS, et al. Incidência e fatores de risco de lesões osteomioarticulares em corredores: Um estudo de coorte prospectivo. Rev Bras Educ Fís Esporte. 2010;24(4):453-62 